\title{
Efecto de la aplicación de diferentes niveles de abono nitrogenado en el cultivo de pimiento (Capsicum annuum) VAR: Yolo Wonder
}

\author{
Effect of the application of different levels of nitrogen fertilizer on the pepper (Capsicum \\ annuum) culture Variety: Yolo Wonder
}

Efeito da aplicação de diferentes níveis de fertilizante nitrogenado na cultura da pimenta (Capsicum annuum) VAR: Yolo Wonder

Kentaro Tomita ${ }^{1,2}$

tomiken30@hotmail.com

Acosta, M. A. S. 1

Vera, P. D. $\mathbf{A}^{\mathbf{1}}$

\begin{abstract}
${ }^{1}$ Universidad Nacional de Pilar - Facultad de Ciencias Agropecuaria \& Desarrollo Rural, Paraguay ${ }^{2}$ Voluntario Senior de JICA: Agencia de Cooperación Internacional del Japón
\end{abstract}

Artículo recibido enero 2018, arbitrado mayo 2018 y publicado en septiembre 2018

\section{RESUMEN}

En la Facultad de Ciencias Agropecuarias, PilarParaguay, se ha realizado una investigación sobre el rendimiento del pimiento"Capsicum annuum" Variedad "Yolo Wonder"; en suelo Entisol (Típico, Psammaquent), con el fin de obtener el máximo rendimiento y estimar la aplicación nitrogenada. Para lograr dicho objetivo se planificó el diseño experimental en bloques al azar, con 5 tratamientos tales como; 0, 50, 100, 150 y $200 \mathrm{Kg}$ de Nitrógeno fraccionadas en tres aplicaciones; aplicadas al momento del trasplante, a los 30,60 días después del trasplante y 4 repeticiones mediante el cual se pudo observar el comportamiento de los niveles aplicados en el estudio, totalizando 20 parcelas. La siembra del pimiento en el lugar definitivo se realizó en hileras a una distancia de $60 \mathrm{~cm}$ entre hileras y $40 \mathrm{~cm}$ entre plantas. La aplicación nitrogenada fue realizada al voleo. De los resultados de análisis de varianza, se observó que hubo diferencia significativa al 1\% en los tratamientos aplicados al cultivo de pimiento en cuanto al número de frutos se refiere, como también al diámetro y el rendimiento de las frutas. En el escenario vegetativo se pudo observar también un alto contenido de Nitrógeno absorbido por la planta, aumentando el nivel de nitrogenado absorbido cuanto mayor fue la aplicación nitrogenada al mismo. Se observó un alto beneficio neto para productores en el tratamiento de $150 \mathrm{kgN} /$ ha sobre estimación económica.

Palabras clave: Aplicación económica; Entisol; Escenario vegetativo; Nitrógeno; Pimiento

\begin{abstract}
On the Faculty of Agricultural Science of the National University of Pilar-Paraguay, it has conducted a research on the Pepper (Capsicum annuum) Variety "Yolo Wonder) Culture in an Entisol soil (Typical, Psammaquent), in order to obtain maximum fruit yield and evaluate the nitrogen application. It was performed the experimental design at randomized blocks with 5 treatments such as: 0, 50, 100, 150 and $200 \mathrm{~kg}$ of Nitrogen divided in three times applications such as at transplanting, 30 and 60 days after transplantation and 4 repetitions by which he could observe the behavior of the application levels on the study, totaling 20 plots. Planting in the final position pepper was performed in rows at a distance of $60 \mathrm{~cm}$ between rows and $40 \mathrm{~cm}$ between plants. The nitrogen application was made at random. From the results of analysis of variance showed that significant difference at $1 \%$ for pepper culture in the number of fruit sand also refers to the diameter and the yield of fruit on the applied treatments. The vegetative stage was also observed a high content of absorbed nitrogen by the plant, increasing the level of nitrogen absorbed the higher nitrogen application at the same time. It was observed the highest net benefit by the application of $150 \mathrm{kgN} / \mathrm{ha}$, it was considered such as the economic application for Pepper Culture.
\end{abstract}

Key words: Economic application; Entisol; Nitrogen; Pepper; Vegetative stage 
RESUMO

Na Faculdade de Ciências Agrícolas, Pilar-Paraguai, foi realizada uma investigação sobre o desempenho da variedade "Capsicum annuum" de pimenta "Yolo Wonder"; no solo Entisol (Típico, Psammaquent), para obter o rendimento máximo e estimar a aplicação de nitrogênio. Para atingir esse objetivo, o delineamento experimental foi planejado em blocos aleatórios, com 5 tratamentos como; 0, 50, 100, 150 e $200 \mathrm{~kg}$ de nitrogênio fracionado em três aplicações; aplicado no momento do transplante, aos 30,60 dias após o transplante e 4 repetições pelas quais foi observado o comportamento dos níveis aplicados no estudo, totalizando 20 parcelas. A semeadura da pimenta no local final foi realizada em fileiras a uma distância de 60 $\mathrm{cm}$ entre as fileiras e $40 \mathrm{~cm}$ entre as plantas. A aplicação de nitrogênio foi realizada difusão. A partir dos resultados da análise de variância, observou-se uma diferença significativa de $1 \%$ nos tratamentos aplicados à cultura da pimenta em termos do número de frutos a que se refere, bem como do diâmetro e rendimento dos frutos. No cenário vegetativo, também foi observado um alto teor de nitrogênio absorvido pela planta, aumentando o nível de nitrogênio absorvido quanto maior a aplicação de nitrogênio nela. Um alto benefício líquido para os produtores foi observado no tratamento de $150 \mathrm{kgN}$ / ha na estimativa econômica.

Palavras-chave: Aplicação econômica; Entisol; Cenário vegetativo; Nitrogênio; Pimentão

\section{INTRODUCCIÓN}

El principal problema en la zona donde se domina en el suelo Entisol en el Departamento de Ñeembucú, es el escaso nivel de producción del cultivo de pimiento Capsicum annuит a nivel comercial, debido principalmente a la baja fertilidad de suelo existente en el distrito de pilar; por lo tanto el productor no toma en consideración que este rubro podría brindar interesantes beneficios económicos (Fatecha, s/f). Esto también puede ser el reflejo del excesivo laboreo de los mismos suelos agrícolas con los cultivos tradicionales como ser el maíz, algodón, mandioca, dos de los cuales necesitan extraer elevados niveles de nutrientes del suelo y en consecuencia se obtiene un bajo rendimiento en el cultivo de pimiento. (Fassbender, 1982, Castagnino, 2008).

Es necesario requerir la bunas técnica para establecer el ssitema dela producción sustentable el fin de aumentar el beneficio neto para productores.

\section{MATERIALES Y MÉTODOS}

\section{Método de investigación}

Para el desarrollo de este estudio se usó el método experimental estadístico (Hernández Sampieri, y otros 2003), el diseño ha sido el de Bloques Completos al Azar. La población y muestra comprende la totalidad de plantas de pimiento en cada una de los bloques, con respecto a la población, y en cuento a la muestra se constituyó por la totalidad de las plantas inscriptas dentro del área útil.

Para la distribución de los tratamientos se diseñó en bloques completamente al azar. Estos fueron cinco tratamientos con cuatro repeticiones o bloques; la distribución de los tratamientos se señala a continuación. 


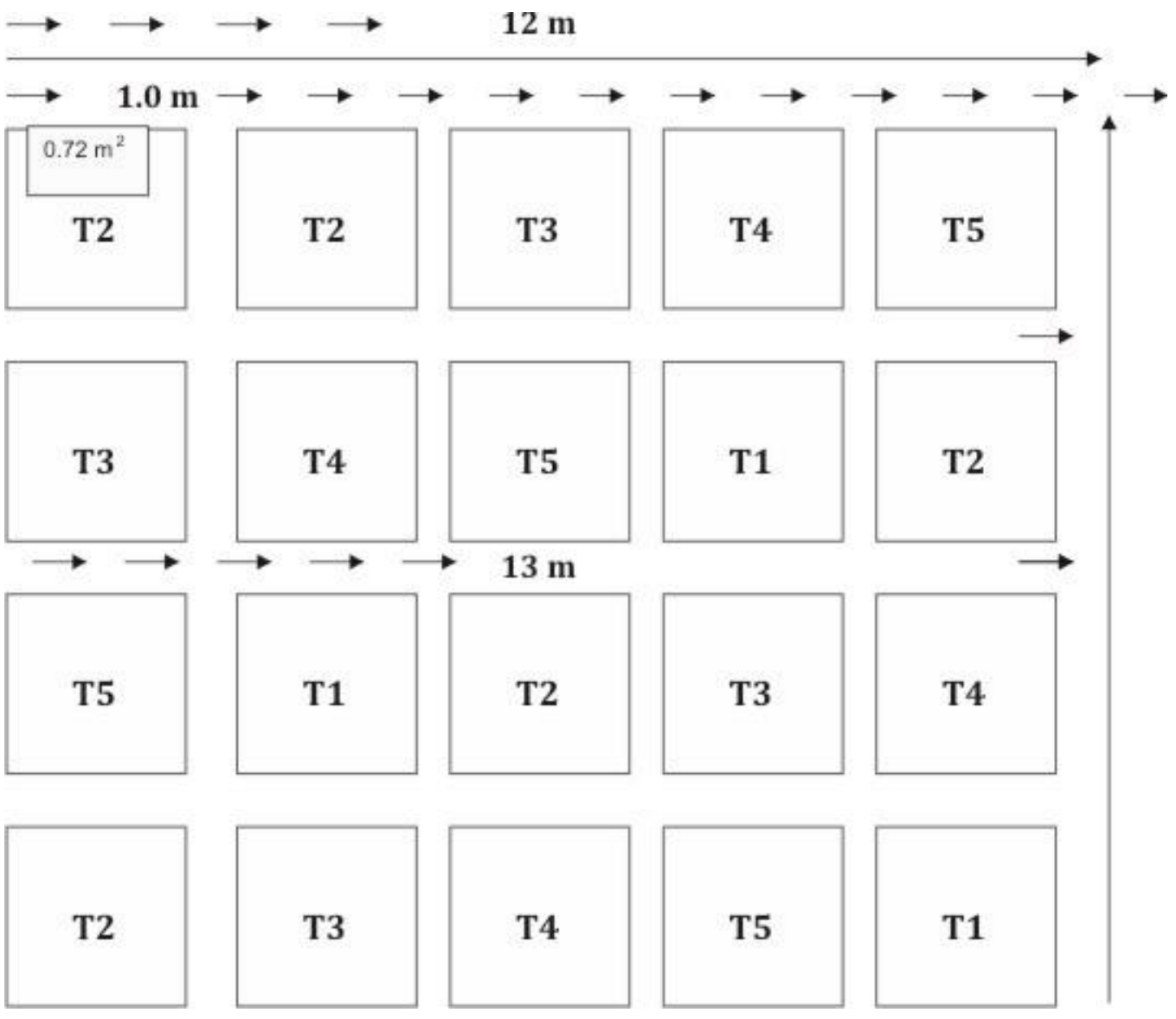

Figura 1. Diseño y dimensiones del experimento.

\section{Observaciones:}

La superficie de cada tratamiento es de 2,4 $\mathrm{m}^{2}$ ( $2 \mathrm{~m}$ de lago por 1,2 $\mathrm{m}$ de ancho).

La distancia entre cada tratamiento es de un metro en cualquier dirección.

Tamaño de la parcela experimental: la superficie del ensayo es de $156 \mathrm{~m}^{2}$ esto es contando con $1 \mathrm{~m}$ más para los bordes de cada lado del terreno, que será objeto de estudio (13 $\mathrm{m}$ de largo por $12 \mathrm{~m}$ de ancho). (Ver la Figura No1).

\section{Las referencias; Tratamientos a aplicar}

Tratamiento № Dosis de Nitrógeno Kg /ha, usando el Urea cuyo contenido 46\% de N.

$\mathrm{T} 1$

$\mathrm{T} 2$

$\mathrm{T} 3$

$\mathrm{T} 4$

$\mathrm{T} 5$
0 Kg de nitrógeno/ha (0 Kg de urea por Hectárea)

$50 \mathrm{Kg}$ de nitrógeno/ha (109 Kg de urea por Ha)

$100 \mathrm{Kg}$ de nitrógeno/ha (217 Kg de urea por $\mathrm{Ha}$ )

$150 \mathrm{Kg}$ de nitrógeno/ha (326Kg de urea por Ha)

$200 \mathrm{Kg}$ de nitrógeno/ha (435 Kg de urea por $\mathrm{Ha}$ ) 


\section{Detalles de la Unidad Experimental}

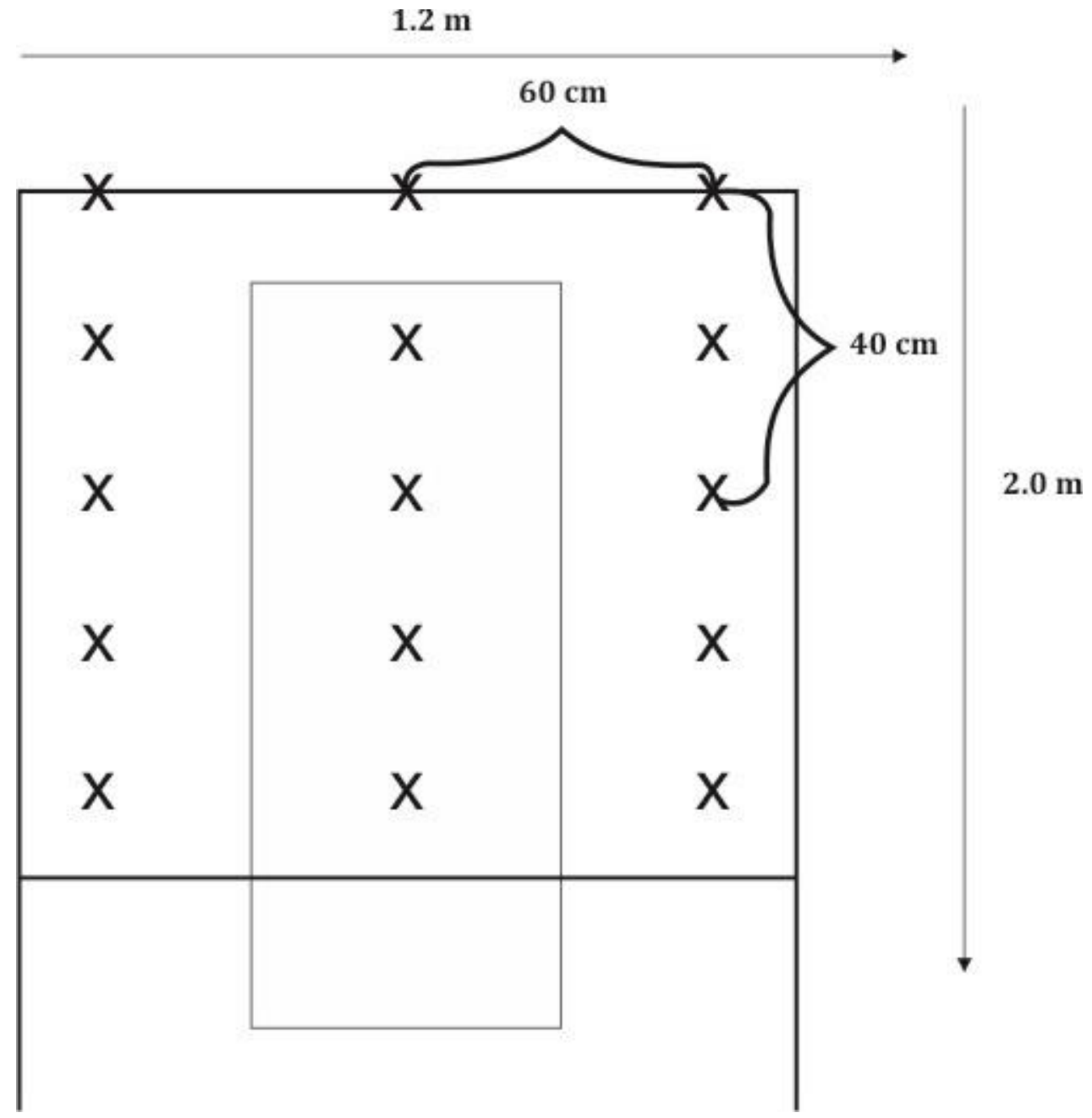

Figura 2. Croquis detallado en el experimento.

La distancia entre plantas es de $0.4 \mathrm{~m}$ entre plantas ( 5 plantas en $2 \mathrm{~m}$ lineal), la distancia entre hileras es de $0.6 \mathrm{~m}$ en cada uno de los tratamientos habrá un total de 15 plantas de pimiento lo que da un total de 300 plantas (población) a utilizar en nuestra parcela experimental. (Ver Figura № 2)

\section{Área útil}

El área útil fue de $0.72 \mathrm{~m}^{2}(0.6 \mathrm{~m} \times 1.2 \mathrm{~m})$, cada parcela contuvo 3 plantas.

Del total de 300 plantas cultivadas; solamente fueron evaluadas un total de 60 plantas (muestra), eliminando las plantas del efecto borde. 


\section{Método del análisis de suelos}

Sobre el análisis de suelos, se tomaron las muestras antes del trasplante, el Prof. Ing. Agr. Kentaro Tomita, PH.D. quien es Coorientador del autor las llevó al Laboratorio de suelos de la fundación Nikkei-CETAPAR (Centro Tecnológico Agropecuario del Paraguay).

En el Laboratorio de la Fundación NikkeiCETAPAR, se utilizó agua destilada (agua: suelo $=1: 1$ ) para determinar el valor de $\mathrm{pH}$, la solución extractora de Mehlich No1 (0.05M $\mathrm{HCl}+0.0125 \mathrm{M} \mathrm{H}_{2} \mathrm{SO}_{4}$ ) para determinar $\mathrm{P}$ y K disponibles, la solución sal de $\mathrm{KCl}$ al $1 \mathrm{M}$ para determinar $\mathrm{Ca}, \mathrm{Mg}$ y $\mathrm{Al}$ intercambiables y el método de Walkley-Black para determinar materia orgánica sobre el análisis de suelos. (Fassbender, 1982).

\section{Método del análisis de tejido vegetal}

Siguiendo lo manifestado por Cáceres Segovia, (1988), el día viernes 3 de febrero de 2012, a los 70 días del cultivo en lugar definitivo y a los 10 días después de la última aplicación de la fertilización nitrogenada se procedió a extraer las muestras para el análisis de tejido vegetal. Dos plantas por tratamiento por bloque. Lo que hace un total de diez plantas por bloque (fuera del área útil); ocho plantas por tratamientos; y fueron extraídos del cultivo finalmente cuarenta plantas siempre fuera del área útil.

Se procedió luego a cortar en pedacitos pequeños con una tijera las ocho plantas de cada tratamiento. Luego se procedió a pesar el papel aluminio que contendría la muestra; se pesa también el peso fresco de la planta de pimiento picado. Esto se lleva a la estufa a una To de 45으 $\mathrm{C}$ por cuatro días para luego volver a pesar y así obtener el peso seco de cada muestra.
Para el análisis de tejido de vegetal se enviaron muestras de los diferentes tratamientos, en la Fundación NikkeiCETAPAR, cuya sede está ubicada en el Distrito Yguazú - Alto Paraná.

En la Fundación Nikkei-CETAPAR se utilizó el método de Kjeldahl para determinar el contenido de $\mathrm{N}$ total $(\mathrm{NH} 4+-\mathrm{N}+\mathrm{NO3-} \mathrm{N}$ ) sobre el análisis de tejido vegetal, usando ácido sulfúrico y ácido salicílico.

\section{RESULTADOS Y DISCUSIÓN}

\section{Característica química del suelo antes de la siembra y la aplicación}

En la siguiente tabla se encuentran los resultados del análisis de suelo antes de realizar el trasplante del pimiento, de los que podemos ver que la medición del pH está en el rango de tolerancia del cultivo, pero se observó bajo contenido de $\mathrm{P}$, por lo que se determinó consecuentemente realizar un ajuste al terreno antes del trasplante al lugar del cultivo. De estos resultados de laboratorio se produce esta investigación de los diferentes niveles de $\mathrm{N}$ en forma de urea, que se podrían utilizar en el cultivo de pimiento para poder alcanzar unos resultados convenientes para el horticultor de la zona estudiada y de zonas con similar cantidad de nutrientes.

Cabe mencionar que el suelo es de tipo arcilloso. (Arcilla de tipo 2:1) aunque también existe una cantidad significativa de arena, lo que tendríamos es un suelo arcillo-arenoso.

También en los resultados de análisis de suelo se puede observar un alto contenido de Ca intercambiable y un bajo contenido de $\mathrm{Al}$ intercambiable; con un muy buen nivel de M.O. para este tipo de suelo (ver la Tabla 1). 
Tabla 1. Característica química del suelo antes del trasplante y la aplicación en los tratamientos

\begin{tabular}{|c|c|c|c|c|c|c|c|c|c|}
\hline \multirow{2}{*}{$\begin{array}{c}\text { Muestra de suelo } \\
\text { Profundidad }\end{array}$} & \multirow{2}{*}{$\begin{array}{c}\text { pH agua } \\
1: 1\end{array}$} & \multicolumn{2}{|c|}{ Disponible } & \multicolumn{4}{|c|}{ Intercambiable } & \multirow[b]{2}{*}{ CICE } & \multirow{2}{*}{$\begin{array}{c}\text { M.O. } \\
\%\end{array}$} \\
\hline & & $\begin{array}{c}\mathrm{P} \\
\mathrm{mg} / \mathrm{kg}\end{array}$ & $\begin{array}{c}\mathrm{K} \\
\mathrm{mg} / \mathrm{kg}\end{array}$ & $\begin{array}{c}\mathrm{K} \\
\mathrm{cmolc} / \mathrm{kg}\end{array}$ & $\begin{array}{c}\mathrm{Ca} \\
\mathrm{cmolc} / \mathrm{kg}\end{array}$ & $\underset{\text { cmolc/kg }}{\mathrm{Mg}}$ & $\begin{array}{c}\text { Al } \\
\mathrm{cmolc} / \mathrm{kg}\end{array}$ & & \\
\hline$(0-20 \mathrm{~cm})$ & 6,03 & 39,3 & 122 & 0,3120 & 4,180 & 1,490 & 0,19 & 6,172 & 2,26 \\
\hline
\end{tabular}

Nota: CICE $=$ Capacidad de Intercambio Catiónico Efectiva $\left(\mathrm{K}+\mathrm{Ca}+\mathrm{Mg}+\mathrm{Al}: \mathrm{cmol}_{\mathrm{c}} / \mathrm{kg}\right)=(0,3120+4,180+$ 1,490 + 0,19: 6,172); M.O. = Materia Orgánica.

\section{Característica agronómica para Pimiento}

La Foto 1 muestra la condición de la cerca de la cosecha para el pimiento en el experimento.

Se ha realizado una investigación sobre el rendimiento del pimiento (Capsicum annuum), Variedad "Yolo Wonder"; con el fin de obtener el máximo rendimiento y estimar la aplicación nitrogenada. Para lograr dicho objetivo se planificó el diseño experimental en bloques al azar, con 5 tratamientos tales como; $0,50,100,150$ y $200 \mathrm{~kg} / \mathrm{N}$ en tres aplicaciones; aplicadas al momento del trasplante, a los 30, 60 días después del trasplante y 4 repeticiones mediante el cual se pudo observar el comportamiento de los niveles aplicados en el estudio, totalizando 20 parcelas. (Material de apoyo de Análisis y Diseño Experimental, s/f).

La siembra del pimiento en el lugar definitivo se realizó en hileras a una distancia de $60 \mathrm{~cm}$ entre hileras y $40 \mathrm{~cm}$ entre plantas. La aplicación nitrogenada fue realizada al voleo. Para otros, se aplicaron $50 \mathrm{kgP}_{2} \mathrm{O}_{5} /$ ha y $50 \mathrm{kgK}_{2} \mathrm{O}$, respectivamente.

En la Figura 3 se muestra la altura de la planta al momento de la cosecha, con respecto a los niveles de Nitrógeno aplicados a lo largo del cultivo; en la figura se muestra la dinámica de la altura de la planta con respecto a las aplicaciones nitrogenadas.

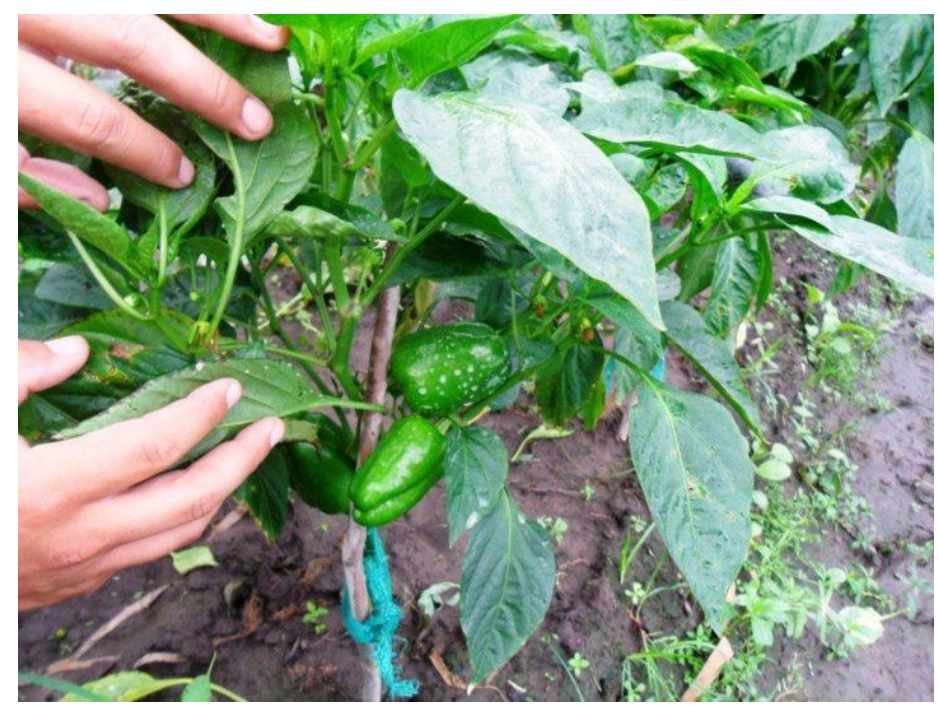

Foto 1. Fruta de Pimiento en el experimento, 2012. 


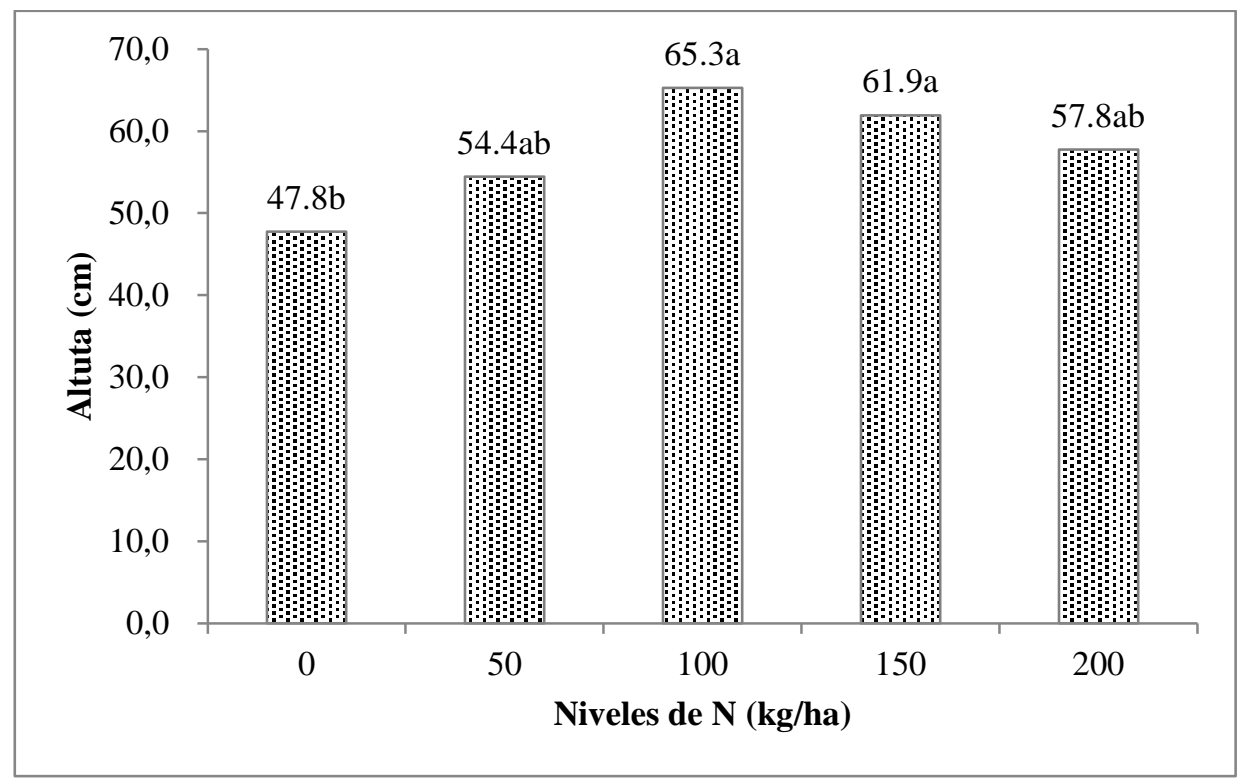

Figura 3. Altura después de la cosecha de acuerdo a la fertilización nitrogenada. (Nota: Medias con la misma letra no son significativamente diferentes en la prueba de rángo múltiple del Duncan).

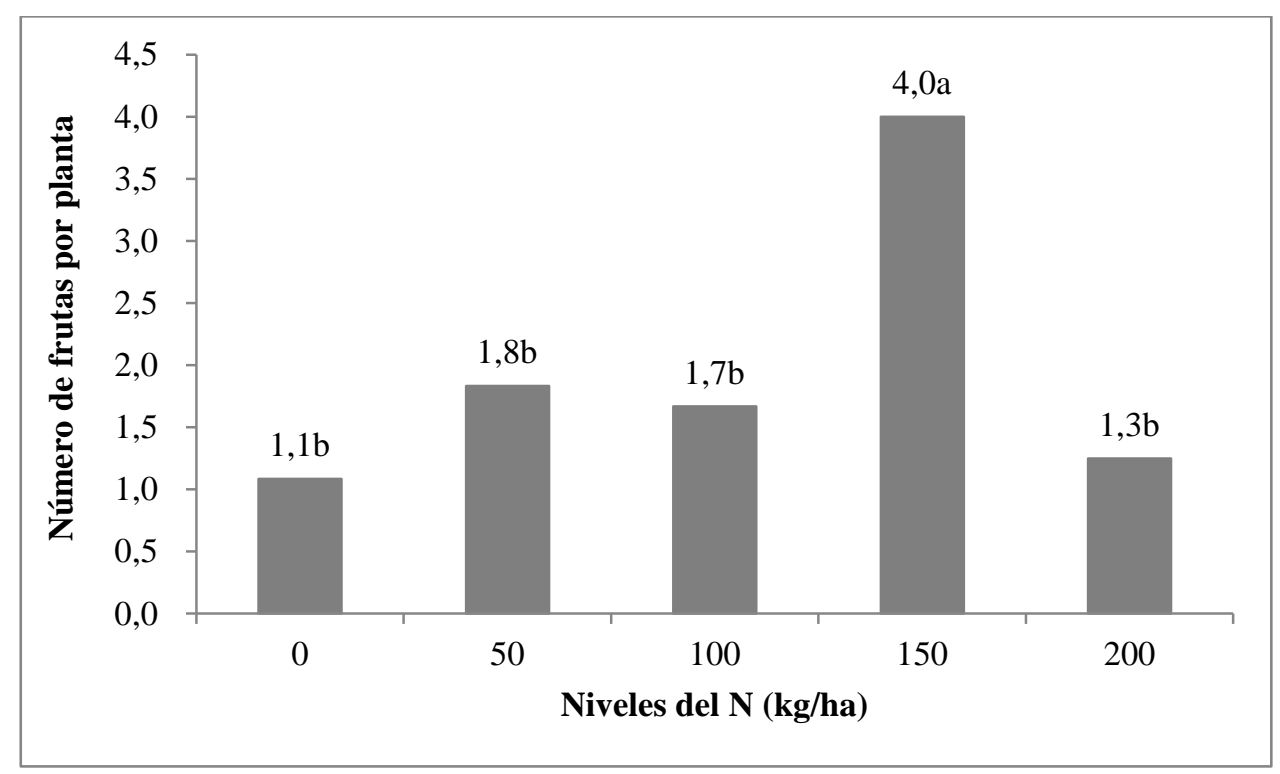

Figura 4. Cantidad de frutas en planta de pimiento de acuerdo a la fertilización nitrogenada. (Nota: Medias con la misma letra no son significativamente diferentes en la prueba de rángo múltiple del Duncan).

La Figura 4 muestra la cantidad de frutos en planta de pimiento de acuerdo a la fertilización nitrogenada. De los resultados de análisis de varianza, se observó que hubo diferencia significativa al $1 \%$ en los tratamientos aplicados al cultivo de pimiento en cuanto al número de frutos y al aplicar $150 \mathrm{kgN} /$ ha el más alto valor.

Alfa, Revista de Investigación en Ciencias Agronómicas y Veterinarias Vol. 2, NNro. 6, Septiembre - Diciembre 2018 
La Figura 5 muestra la longitud de fruta en cada tratamiento nitrogenado. De los resultados de análisis de varianza, se observó la diferencia significativa al 5\% para el tratamiento y fue el más alto para el tratamiento con $100 \mathrm{kgN} / \mathrm{ha}$.

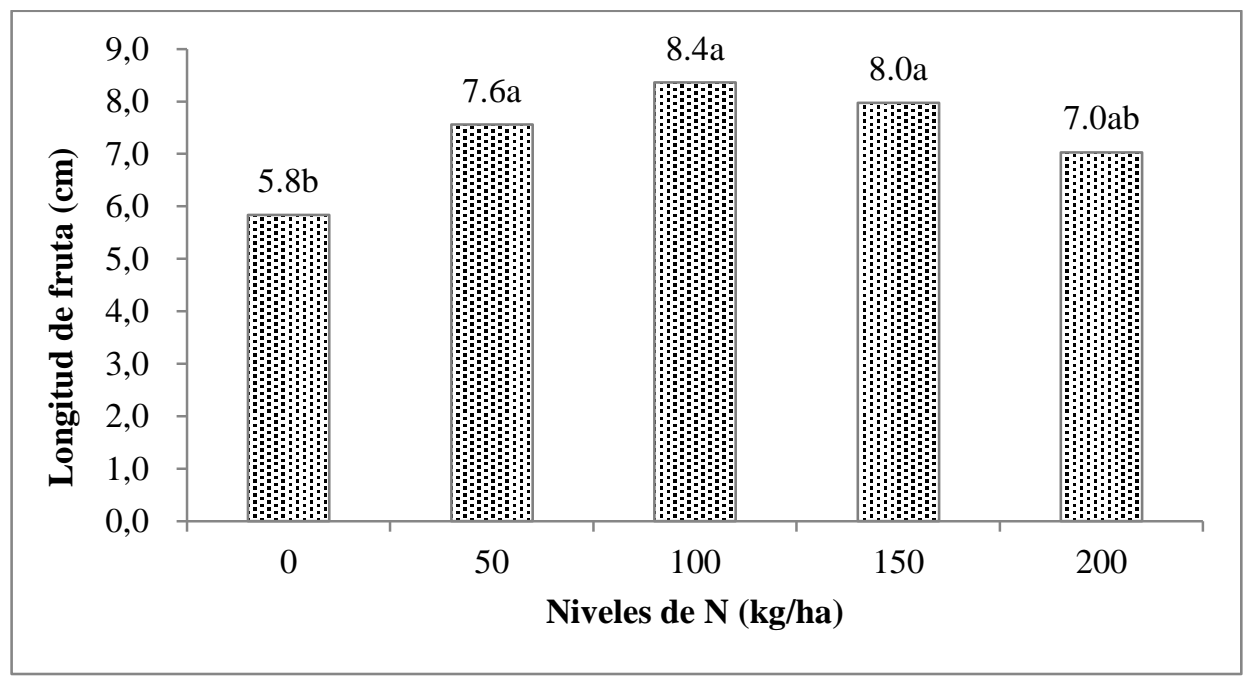

Figura 5. Altura de frutos en planta de pimiento de acuerdo a la fertilización nitrogenada. (Nota: Medias con la misma letra no son significativamente diferentes en la prueba de rángo múltiple del Duncan).

A continuación, la Figura 6 muestra el diámetro de la fruta en cada tratamiento. De los resultados de análisis de varianza, se observó la diferencia significativa al $1 \%$ y para el tratamiento con $100 \mathrm{kgN} / \mathrm{ha}$ el más alto ai igual que el caso de la longitud.

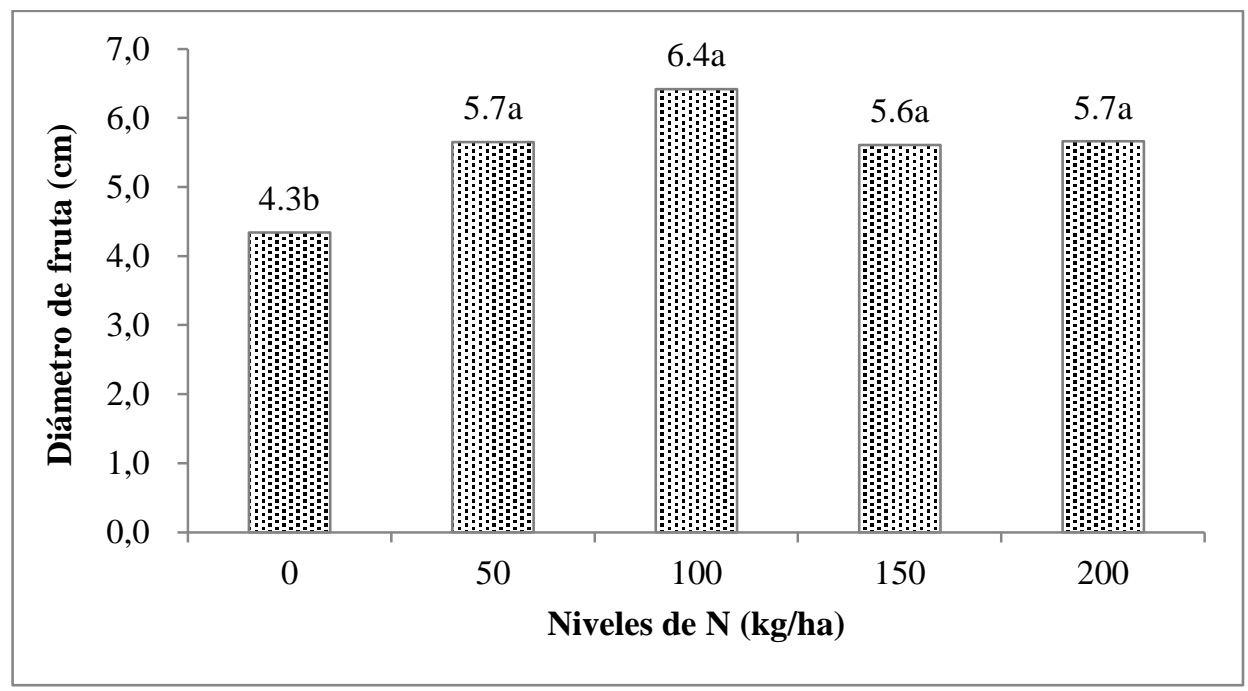

Figura 6. Diámetro de frutos en planta de pimiento de acuerdo a la fertilización nitrogenada. (Nota: Medias con la misma letra no son significativamente diferentes en la prueba de rángo múltiple del Duncan). 


\section{Rendimiento de la fruta para Pimiento}

En la Figura 7 se muestra la dinámica del rendimiento de las frutas de pimiento existente al momento de la cosecha con respecto a los niveles del $\mathrm{N}$ aplicados a lo largo del cultivo en cada tratamiento. De los resultados de análisis de varianza, se observó que hubo diferencia significativa al $1 \%$ en los tratamientos aplicados al cultivo de pimiento en cuanto al rendimiento al igual que el número de frutos. Con la evaluación económica para el cultivo de pimiento, al aplicar $150 \mathrm{kgN} / \mathrm{ha}$, se observó un alto beneficio neto para productores.

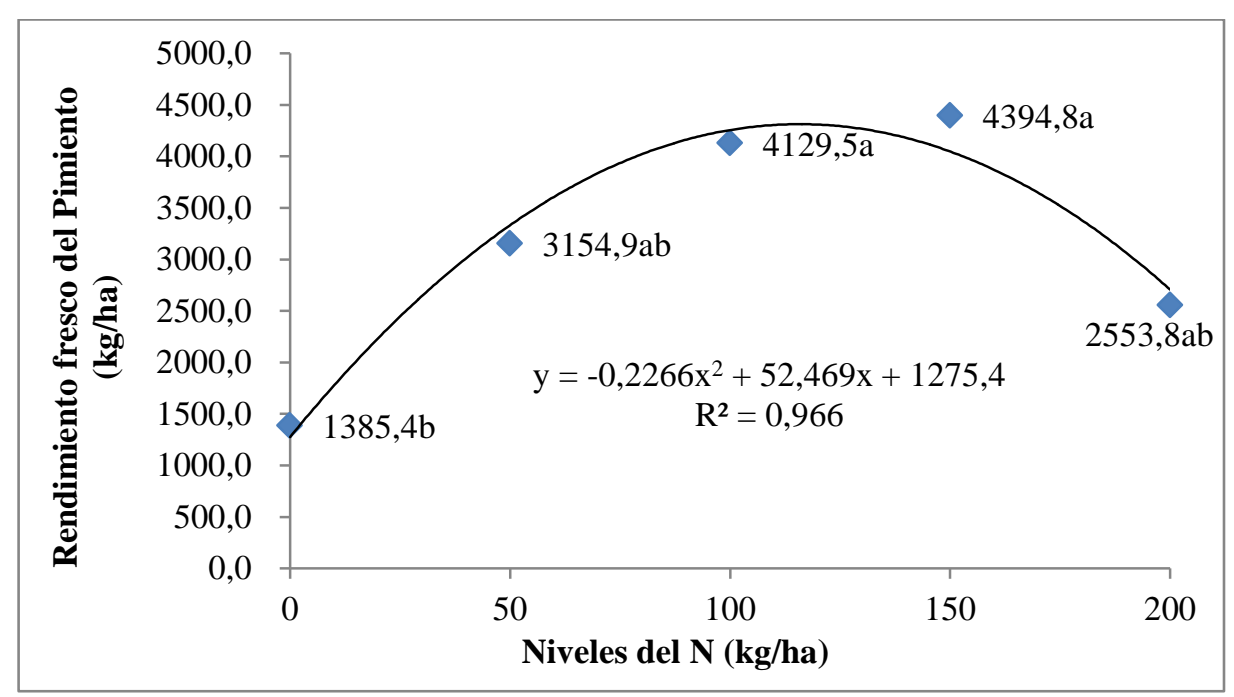

Figura 7. Dinámica del rendimiento de la fruta de pimiento de acuerdo a la fertilización nitrogenada. (Nota: Medias con la misma letra no son significativamente diferentes en la prueba de rango múltiple del Duncan).

En el escenario vegetativo se pudo observar también un alto contenido de nitrógeno absorbido por la planta, aumentando el nivel de nitrogenado absorbido cuanto mayor fue la aplicación nitrogenada al mismo (ver la Figura 8). Se observó alta relación entre la absorción nitrogenada en el escenario vegetativo y el rendimiento de la fruta, es muy importante que realicemos el manejo del $\mathrm{N}$ para el cultivo. 


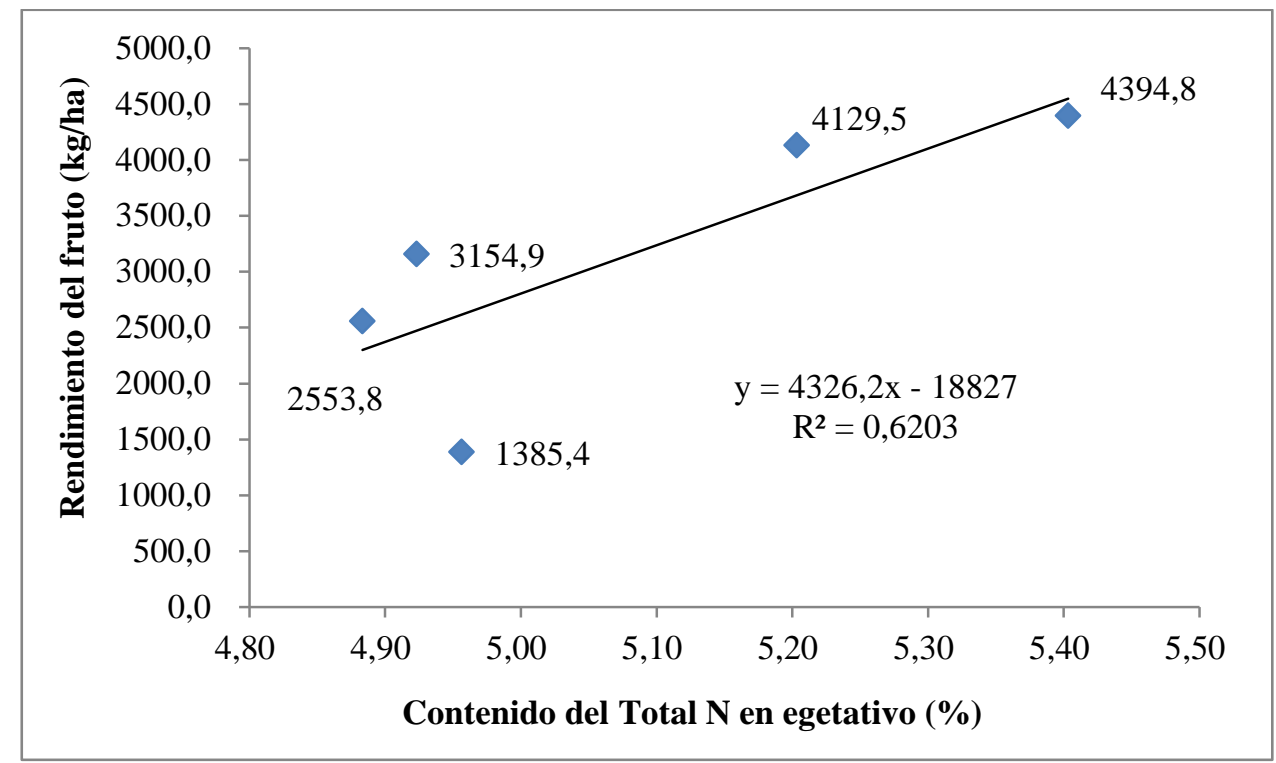

Figura 8. Dinámica de la relación del nitrógeno en el escenario vegetativo y fruta de pimiento de acuerdo a la fertilización nitrogenada.

\section{Evaluación económica para el cultivo de Pimiento}

La Tabla 2 muestra el costo fijo para el cultivo de pimiento. Además, la Tabla №3 muestra la evaluación económica para el cultivo de pimiento. Para el rendimiento de la fruta, no se observó la gran diferencia entre 100 y $150 \mathrm{kgN} / \mathrm{ha}$. Con el resultado de evaluación económica y número de fruta por planta, el fue más alto para el tratamiento con $150 \mathrm{kgN} / \mathrm{ha}$.

Tabla 2. Costo fijo para el cultivo de pimiento

\begin{tabular}{lccc}
\hline Costo fijo \\
\hline \multicolumn{4}{l}{ Preparación del terreno } \\
\hline Actividades & Cantidad & Precio Unitario & Costo total (US\$) \\
Arada & 1 & 48 & 48 \\
Rastreada & 1 & 36 & 36 \\
\hline \multicolumn{4}{c}{ Sub total en guaraníes } \\
\hline
\end{tabular}

\begin{tabular}{cccccc}
\hline Mano de obra & \multicolumn{7}{c}{} & \\
\hline Actividades a realizar & Cantidad & Dias de Trabajo & № de personales & $\begin{array}{c}\text { Precio } \\
\text { jornal }\end{array}$ & $\begin{array}{c}\text { Costo } \\
\text { total(US\$) }\end{array}$ \\
\hline Almácigo & 1 & 1 & 1 & 10 & 10 \\
Siembra & 1 & 1 & 1 & 10 & 10 \\
Traplante & 1 & 1 & 2 & 10 & 19 \\
Carpida & 1 & 11 & 2 & 10 & 19 \\
Riego & 1 & 22 & 1 & 10 & 10 \\
Cosecha & 1 & 1 & 1 & 10 & 10 \\
\hline
\end{tabular}

Alfa, Revista de Investigación en Ciencias Agronómicas y Veterinarias Vol. 2, NNro. 6, Septiembre - Diciembre 2018 


\begin{tabular}{lccr}
\hline Insumo & & & \\
\hline Descripcion & Cantidad(g/Ha) & Precio(US $\$ / g)$ & $\begin{array}{c}\text { Costo total } \\
(\text { US } \$ / \text { ha })\end{array}$ \\
\hline Semillas & 500 & 0,114 & 57 \\
Descripcion & Cantidad(L/Ha) & Precio & Costo total \\
Glifosato & 5 & 9 & 44 \\
\hline \multicolumn{1}{c}{ Total Costo en guaraníes } & Sub total en guaraníes & 101 \\
\hline
\end{tabular}

Tabla 3. Evaluación económica para el cultivo de pimiento.

\begin{tabular}{|c|c|c|c|c|c|c|c|c|}
\hline $\begin{array}{c}\mathrm{N} \\
\mathrm{Kg} / \mathrm{Ha}\end{array}$ & $\begin{array}{l}\text { Rendimiento } \\
\text { estipulado } \\
\text { (kg/ha) }\end{array}$ & $\begin{array}{c}10 \% \\
\text { perdida } \\
\text { en la } \\
\text { cosecha } \\
\text { (kg/ha) }\end{array}$ & $\begin{array}{l}\text { Kg a ser } \\
\text { vendida } \\
\text { ( } \mathrm{kg} / \mathrm{ha})\end{array}$ & $\begin{array}{c}\text { Beneficio } \\
\text { bruto. } \\
\text { (US\$ /ha) }\end{array}$ & $\begin{array}{c}\begin{array}{c}\text { Costo } \\
\text { variable }\end{array} \\
\text { (US\$ } \\
\text { /ha) } \\
\end{array}$ & $\begin{array}{c}\begin{array}{c}\text { Costo } \\
\text { fijo }\end{array} \\
\text { (US\$ } \\
\text { /ha) } \\
\end{array}$ & $\begin{array}{c}\text { Costo } \\
\text { total }\end{array}$ & $\begin{array}{c}\begin{array}{c}\text { Beneficio } \\
\text { neto }\end{array} \\
\text { (US\$ /ha) }\end{array}$ \\
\hline 0 & 1385,4 & 138.54 & 1246.86 & 1.930 & 0 & 568 & 568 & 1.362 \\
\hline 50 & 3154,86 & 315,48 & 2839,38 & 4.394 & 81 & 568 & 649 & 3.745 \\
\hline 100 & 4129,5 & 412.95 & 3716.55 & 5.752 & 162 & 568 & 730 & 5.022 \\
\hline 150 & 4394.80 & 439.48 & 3955.32 & 6.121 & 243 & 568 & 811 & 5.310 \\
\hline 200 & 2553.82 & 255.38 & 2298.44 & 3.557 & 324 & 568 & 892 & 2.665 \\
\hline
\end{tabular}

Nota: Precio del pimiento al momento de la cosecha fue de US\$1.55 Gs $/ \mathrm{kg}$, Costo del Nitrógeno por Kilogramo fue de US $\$ 1.62$ (Costo de la urea fue de US $\$ 37.3 / 50 \mathrm{~kg}$ ) y el costo fijo fue de US\$160/ha.

\section{CONCLUSIONES}

Con relación al número de frutas por planta, podemos concluir que hubo diferencia altamente significativa en el cuadro de análisis de varianza (Anova) (Ver la Figura 4) y en la prueba de comparación de rango múltiple de Duncan demostró que el tratamiento número cuatro $(150 \mathrm{Kg} \mathrm{N} / \mathrm{ha})$, es altamente superior a los tratamientos número uno (0 Kg N/ha), cinco (200Kg N/ha), tres $(100 \mathrm{Kg} \quad \mathrm{N} / \mathrm{ha}) \quad \mathrm{y} \operatorname{dos}(50 \mathrm{Kg} \quad \mathrm{N} / \mathrm{ha})$ respectivamente para el cultivo de pimiento variedad Yolo Wonder.

Con relación al rendimiento de las frutas, podemos indicar que hubo diferencia significativa en el cuadro de análisis de varianza (Anova) (Ver la Figura 7) y en la prueba de comparación de rango múltiple de Duncan demostró que el tratamiento número cuatro $(150 \mathrm{Kg} \mathrm{N} / \mathrm{ha})$, es significativo con respecto a los tratamientos número uno $(0 \mathrm{Kg}$ $\mathrm{N} / \mathrm{ha}$ ) y cinco (200Kg N/ha) respectivamente; también podemos apreciar que los tratamientos número tres (100 Kg N/ha) y dos (50 Kg N/ha) son significativos con respecto al tratamiento número uno $(0 \mathrm{Kg}$ $\mathrm{N} / \mathrm{ha}$ ) en el rendimiento de la fruta de pimiento variedad Yolo Wonder en $\mathrm{Kg} / \mathrm{ha}$.

Con relación a la estimación económica del cultivo de pimiento variedad Yolo Wonder, se concluye que el tratamiento número cuatro (150 $\mathrm{Kg} \mathrm{N} / \mathrm{ha}$ ) brindó el mayor beneficio neto con US\$5310/ha. El mismo fue el precio más alto obtenido para la 
rentabilidad económica en comparación con los demás tratamientos. (Ver la Tabla 3).

\section{REFERENCIAS}

Cáceres Segovia, F. (1988). Apunte de horticultura. Facultad de Ingenieria Agronómica. U.N.A. Asunción Paraguay. $102 \mathrm{p}$

Castagnino, A. (2008). Manual de cultivos hortícolas innovadores. $1^{\circ}$ ed. Buenos Aires, Ar. Hemisferio sur. 260p

Fassbender, H. (1982). Química de suelos con énfasis en suelos de América Latina. $1^{\circ}$ Edición. San José, Costa Rica. IICA. 398 p

Fatecha, A. (s/f). Fertilidad de suelo. Quinto semestre. Material de apoyo de Olericultura del $3^{\circ}$ Año de la carrera de
Ingeniería Agropecuaria de la Facultad de Ciencias Agropecuarias y D.R

Hernández Sampieri, R.; Fernández, C. y Batista, L. (2003). Metodología de la investigación. $3^{\circ}$ ed. México D.F. McGrawHill. $705 \mathrm{p}$

Material de apoyo de Análisis y Diseño Experimental del 4읏 Año de la carrera de Ingeniería Agropecuaria de la Facultad de Ciencias Agropecuarias y Desarrollo Rural

Material de apoyo de Metodología de la investigación I y II del 4ํㅡㅁono de la carrera de Ingeniería Agropecuaria de la Facultad de Ciencias Agropecuarias y Desarrollo Rural 